to conclude that there is need for considerable caution in the interpretation of such observations.

In a previous communication ${ }^{1}$, the unsuitability of the white rat as a host for this purpose was suggested, for though the course of an infection in cotton rats and white rats follows the same pattern, in the white rat the microfilariæ do not persist in the circulation for the same length of time as in the cotton rat, and the death of the parent worms and their encapsulation occurs much earlier. Though the infection in the cotton rat has the same evolution, there is less disparity between its duration and the life span of the host. Nevertheless a cotton rat infected in its youth may outlive an infection which has undergone spontaneous cure. We have been able to follow, for a year or more, the general course of the blood infection in cotton rats exposed to infection once-usually for 24 hours-by small numbers of infective mites. Microfilariæ appear in the peripheral blood on the fifty-first day after exposure, and increase during the next month or more, after which the count tends to remain more or less constant, with wide variations about the mean. In one rat, still surviving, an infection intensity, varying between 140 and 240 microfilariæ/c.mm., has been maintained for the past twelve months. On the other hand, in another rat, the general level, varying between 80 and 190 microfilariæ/c.mm., persisted for some six months; but, during the following three or four months, the count dropped progressively until the microfilariæ finally disappeared three weeks before the animal's death, when only dead encapsulated worms were found.

In other cotton rats, infected on several occasions by mass exposure to mites, the infection has followed a similar course. Some, which showed counts of 1,500 , $840,675,450$ and 150 microfilariæ/e.mm. in the peripheral blood about the third or fourth month after infection, dropped after the seventh or eighth months to $530,45,30,10$ and 20 microfilariæ/c.mm. respectively, while in others, which died seven to twelve months after their last exposure, many of the adult worms were found to be dead and encapsulated.

The relationship between the death of the adult worms and the disappearance of the microfilariæ from the peripheral circulation cannot be defined in the absence of more precise knowledge of the migrationrate and the longevity of the microfilariæ. Though they are found most commonly in the peripheral circulation within one day of injection into the pleural or peritoneal cavities and the subcutaneous tissue, their persistence in the circulation in the absence of the adult varies from days to weeks and even months. Furthermore, in one such animal, a concomitant septic infection has been accompanied by a transient reappearance of the circulating microfilariæ after they had previously disappeared for a time, suggesting that redistribution may be an additional factor in their decline.

Our results cannot be taken to indicate the minimum time required before gross pathological changes are induced in the pleural sac by the adult worms, nor do they enable the duration of a particular blood infection to be estimated before the progressive and terminal fall occurs in the microfilarial count, nor can the disappearance of the microfilariæe and the death of the adult worm be correlated accurately at the moment. Nevertheless, they suggest that conclusions from treated infections should be made with due regard to the findings in untreated controls, and that results derived from wild, naturally infected cotton rats should be interpreted with caution.

W. E. KerRShaW

D. S. Bertram

Department of Entomology and Parasitology, School of Tropical Medicine, Pembroke Place, Liverpool 3. April 12.

${ }^{1}$ Nature, 158, 418 (1946).

\section{Density of the Blackfish Petrosal}

THE mammalian petrosal is generally recognized by anatomists as the most compact of bones. The physical density of the various bones does not seem to have attracted much attention. The only data in the literature available to me are those of the "Smithsonian Physical Tables", where the density of bone is given as varying from 1.2 to $2.0 \mathrm{gm}$. per cubic centimetre.

Mr. J. T. Sorenson included a petrosal from a blackfish (Globicephala melcena) in the collection which he made in his second stay at Campbell Island in the New Zealand subantarctic group. The extraordinary density of this specimen is striking and immediately apparent when the bone is handled. Although the specimen measures only $40 \mathrm{~mm}$. $\times$ $28 \mathrm{~mm}$. $\times 18 \mathrm{~mm}$. in its major dimensions, the dry weight is $\mathbf{1 7 . 5} \mathrm{gm}$. After boiling in water to expel air, the volume measured by imrnersion is $5 \cdot 0$ c.c.; accordingly, the physical density is $3 \cdot 5$, considerably exceeding the range given in the Smithsonian "Physical Tables".

In comparison, the petrosal of a dog weighed 1.34 gm. and had a volume, measured as before, of $1 \cdot 0$ c.c. The density of this specimen, $1 \cdot 34$, falls well within the limits given in the tables, and confirms the initial impression of the remarkable density of the blackfish petrosal.

Department of Zoology,

Victoria University College,

Wellington, New Zealand.

\section{A Method for Studying the Carbohydrate Metabolism of Micro-organisms}

WE wish to emphasize the usefulness of the method of paper chromatography of sugar ${ }^{1,2}$, especially when applied on a quantitative basis ${ }^{3}$, in the study of bacterial metabolism. As an example we quote the following experiment on the utilization of sucrose by Leuconostoc mesenteroides (Lister Institute strain No. 3351 ).

Most strains of this bacterium are found to produce copious yields of a dextran from this sugar and no other4. The strain used had those properties.

During the production of the gum, large quantities of reducing sugars are formed, and it has been generally assumed that the glucose part of the sucrose is polymerized to dextran with simultaneous liberation of the fructose moiety ${ }^{5}$. Considerable interest is centred on the bacterium due to Hehre and Sugg's ${ }^{6}$ enzymatic synthesis of the dextran.

A loopful of a 48-hr. culture of the organism (growing in Tarr and Hibbert's liquid medium ${ }^{4}$ with the addition of 2 per cent yeast extract) was inoculated into $10 \mathrm{ml}$. of this medium, and the culture flask incubated at $28^{\circ} \mathrm{C}$.

It is possible by the paper chromatography method to estimate at any desired interval during growth the 